\title{
'Pee'BuSt Trial: A single-centre prospective randomized study comparing functional and anatomic outcomes after augmentation urethroplasty with penile skin graft versus buccal mucosa graft for anterior urethral stricture disease
}

\author{
Shantanu Tyagi ${ }^{1} \cdot$ Kalpesh Mahesh Parmar $^{1}$ (1) - Shrawan Kumar Singh ${ }^{1} \cdot$ Anuj Sharma ${ }^{1} \cdot$ Mukesh Shukla $^{2}$. \\ Aditya Prakash Sharma ${ }^{1}$. Sudheer Kumar Devana ${ }^{1}$. Gopal Sharma ${ }^{1}$ - Santosh Kumar ${ }^{1}$. Arup Kumar Mandal ${ }^{1}$
}

Received: 9 July 2021 / Accepted: 18 September 2021 / Published online: 16 October 2021

(c) The Author(s), under exclusive licence to Springer-Verlag GmbH Germany, part of Springer Nature 2021

\begin{abstract}
Purpose To assess the surgical outcomes of augmentation urethroplasty (AU) using penile skin graft (PSG) compared to buccal mucosa graft (BMG) in anterior urethral stricture disease.

Method Between January 2018 and January 2019, 100 patients with anterior urethral stricture planned for AU were randomized into PSG or BMG arms (CTRI/2018/07/015028). Anatomic and functional variables were compared pre-operatively and post-operatively. Primary outcome was success rate at 18 months and it was defined if any of the three criteria were met, i.e. either maximum urinary flow (Qmax) $>15 \mathrm{ml} / \mathrm{s}$ or urethral calibration of 16 French or ability to traverse the repair with 17 French cystoscope. Secondary outcomes were functional parameters such as International Prostate Symptom Score (IPSS), International Index of Erectile Function (IIEF) Score, Male Sexual Health Questionnaire for Ejaculatory Dysfunction (MSHQ-EJD), and Urethral Stricture Surgery-Patient Related Outcome Measure (USS-PROM).

Results Pre-operative variables were comparable between both the arms. Median duration of follow-up was 22 months (18-24 months). At 18 months, the success rates of AU with PSG and BMG were comparable (89\% v/s 91\%; $p=0.70$, $95 \%$ CI- 0.33 to 5.21). The improvements in Qmax $(p=0.06)$, IPSS $(p=0.43)$ and USS-PROM $(p=0.49)$ were comparable between the two arms. There was no statistically significant difference in the IIEF-Erectile domain $(p=0.07)$, IIEF-Orgasmic domain $(p=0.11)$ and MSHQ-EJD $(p=0.20)$ following AU at 18 months. Clavien-Dindo grade I complications were $12.7 \%$ in PSG and $16.7 \%$ in BMG.

Conclusion This study provides level 1 evidence of no statistical significant difference in outcomes of AU using BMG or PSG.
\end{abstract}

Keywords Buccal mucosa graft $\cdot$ Penile skin graft $\cdot$ Augmentation urethroplasty $\cdot$ Urethral stricture $\cdot$ LUTS $\cdot$ PROMS

\section{Introduction}

Augmentation urethroplasty (AU) is traditionally best suited for the management of non-obliterative long segment anterior urethral strictures. It is being performed using various

Kalpesh Mahesh Parmar

kalpesh010385@gmail.com

1 Department of Urology, Post Graduate Institute of Medical Education and Research (PGIMER), Advanced Urology Ward, Level 2, B-Block, Chandigarh, Punjab, India 160012

2 Department of Community Medicine, Veer Chandra Singh Garhwali Government Institute of Medical Science \& Research, Srinagar, Uttarakhand, India types of grafts, each having their own benefits and limitations. These grafts can be fashioned from locally available genital skin, preferably hairless (prepuce, distal penile and inguinal region) or from distant mucosal sites (buccal, lingual, bladder, intestinal mucosa, saphenous vein) or more recently from "tissue engineered" templates [1].

Buccal mucosal graft (BMG) is the most commonly used graft due to its characteristic advantages. However, BMG may not be available in conditions like mucosal fibrosis, mucosal ulcerations, oral infections, oral pre-malignant and malignant lesions. The other alternative is penile skin graft (PSG) or Inner preputial skin graft (IPG), which is easy to handle, familiar to the urologist and available locally in the operating field. It can be harvested under the same regional 
anaesthesia used for urethroplasty and therefore morbidity of general anaesthesia can be avoided, especially in highrisk patients.

When multiple options are available, the question remains as to which is the best graft material in terms of success and morbidity [1]. Though traditionally, superior outcomes have been reported with BMG over PSG [2], this can be settled only by level 1 evidence, but still to date no well-designed randomised study has been published in this regard. We conducted this randomised study to compare the outcomes of augmentation urethroplasty performed with either BMG or PSG.

\section{Methods}

This is a single tertiary care centre, parallel design randomized controlled trial named 'Pee'BuSt trial, "Pee" stands for penile skin, "Bu" for buccal, and "St" for substitution/ augmentation urethroplasty. The allocation ratio for this study was $1: 1$. This study was registered with ctri.nic.in, Clinical Trial registry of India (CTRI/2018/07/015028).

Patients presenting to our outpatient clinic with anterior urethral stricture, who were scheduled for AU, were enrolled in our study. Patients were included if they had an anterior urethral stricture greater than $2 \mathrm{~cm}$. Patients with balanitis xerotica obliterans, urethrocutaneous fistula, scarred perineum, unhealthy/unavailable buccal mucosa or preputial skin were excluded. The study protocol, informed consent, patient information document and data collection forms were approved by the Institutional Ethics Committee (INT/ EC/2018/000909).

Pre-operative functional or subjective assessment included International Prostate Symptom Score (IPSS) [3] for lower urinary tract symptoms (LUTS), International Index of Erectile Function (IIEF) Score [4], Male Sexual Health Questionnaire for Ejaculatory Dysfunction (MSHQEJD) [5], Urethral Stricture Surgery-Patient Related Outcome Measure (USS-PROM) [6], which is a combination of LUTS domain, Peeling's voiding picture [7], a generic health status domain (EQ-5D-3L of the EuroQol group) [8], and a treatment satisfaction question (Supplementary file).

Anatomic or objective assessment included calibration of the urethra, uroflowmetry (UFM), retrograde urethrography (RGU) and voiding cystourethrography (VCUG). Using 6/7.5 French semirigid ureteroscope, intra-operative assessment of urethral calibre and urethral mucosa was done. The "dorsal onlay" graft placement was performed for AU [9] (operative technique; Supplementary file).

The urethral catheter was removed at 3 weeks after surgery. Peri-catheter RGU was done in all patients at the time of catheter removal. Subsequent follow-up was performed at
3, 6, and 12 months and bi-annually thereafter. Patients were advised to report earlier if necessary. Surgical complications were graded according to Clavien-Dindo classification.

Primary outcome, i.e. success rate was evaluated at 18 months [4] and it was defined by a maximum urinary flow $(\mathrm{Qmax})>15 \mathrm{ml} / \mathrm{s}$. If Qmax $\leq 15 \mathrm{ml} / \mathrm{s}$, urethral calibration admitting 16 French Foley catheter was considered as success. If urethral calibration with 16 Fr Foley catheter was not successful, an ability to traverse the repair with 17 French cystoscope was considered as success. AU was counted as success if any of the three criteria were met, i.e. either maximum urinary flow $(\mathrm{Qmax})>15 \mathrm{ml} / \mathrm{s}$ or urethral calibration of 16 French or ability to traverse the repair with 17 French cystoscope. Any intervention including dilatation or visual internal urethrotomy (VIU) in the post-operative period was considered failure. Secondary outcomes were functional parameters such as IPSS, IIEF, MSHQ-EJD and USS-PROM and the association of variables such as length, aetiology and site of urethral stricture affecting the success rate of $\mathrm{AU}$.

Based on standard literature review [4], for 5\% level of significance and for $80 \%$ power of the study, the calculated sample size was 44 in each arm. Estimating a $10 \%$ attrition rate, 100 patients were randomized into two arms. Block randomization with computerised random number generator was used to assign sample numbers equally to each arm, with every single block comprising four items. Allocation was concealed in a sealed envelope and was revealed by an operating room technician once the patient was shifted to the operating table. The procedures were done by two trained and experienced surgeons.

The "statistical analysis" was done using Statistical Package for Social Sciences "SPSS Inc., Chicago, IL, version 23.0 for Windows" and Stata (StataCorp. 2015. Stata Statistical Software: Release 14. College Station, TX: StataCorp LP). Proportions were matched using 'Chi-square or Fisher's exact test' and $t$ test was used for comparisons of mean between two arms. Each statistical checks were "two-sided" and were implemented at a "significance level of $a \leq 0.05$ " (detailed statistical analysis plan; CONSORT checklist; Supplementary file).

\section{Results}

A total of 170 patients with anterior urethral stricture were assessed for eligibility from January 2018 to January 2019 at our tertiary care centre. Complying with inclusion and exclusion criteria, 100 patients were randomised into two arms. At 18 months, we analysed the data of 47 patients in the PSG/IPG and 48 in the BMG arm (Fig. 1). Preoperative variables such as age, BMI, duration of stricture, length of stricture, aetiology and pre-operative interventions 
Fig. 1 Consort diagram for the study

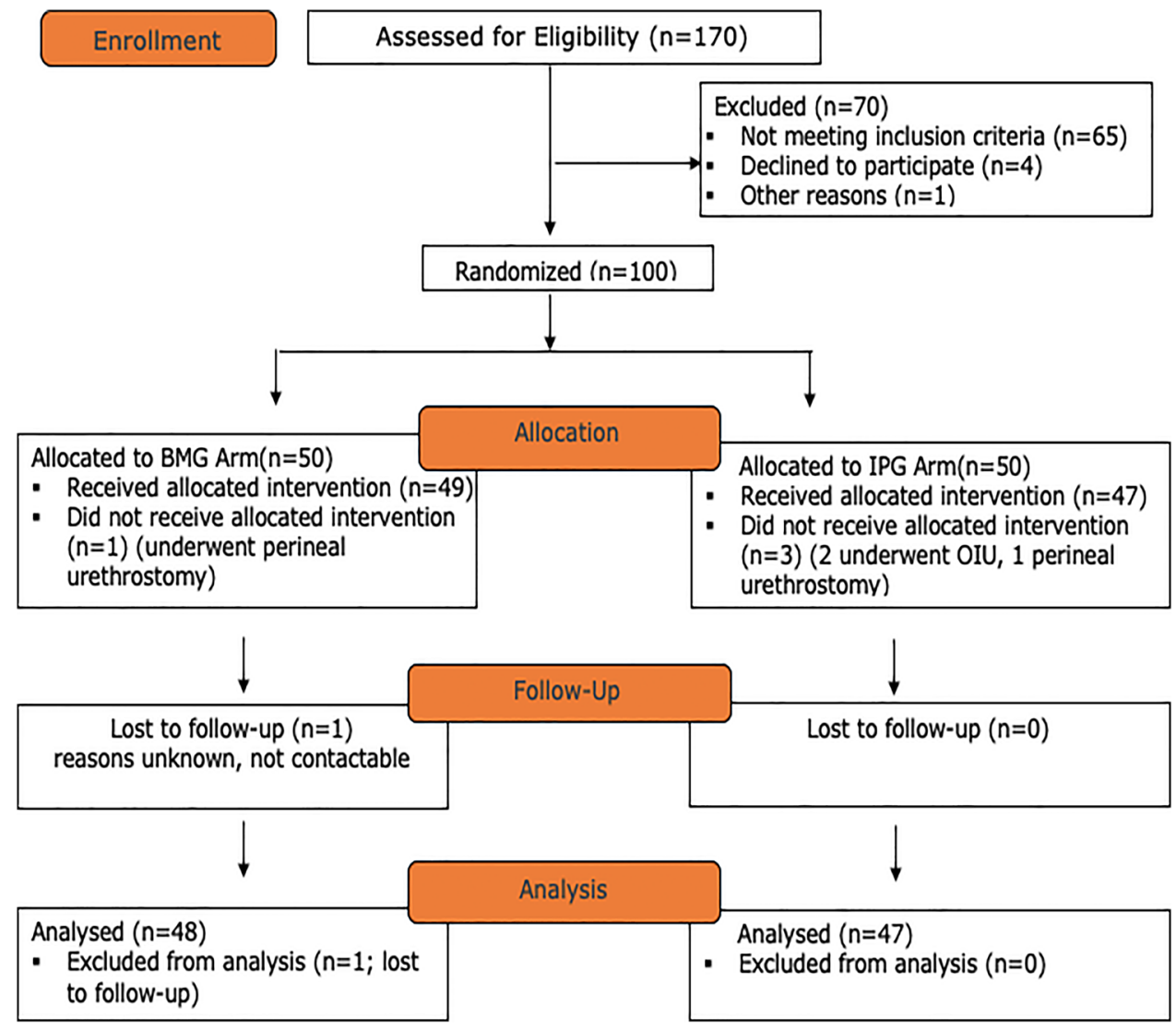

were comparable between both the arms (Table 1). Overall, the median stricture length was $55 \mathrm{~mm}$ and in majority, $(70 \%)$ stricture involved the peno-bulbar region. Graft from bilateral cheeks were required in $31 \%$ (15/48) patients in the BMG arm. The median duration of follow-up was 22 months (interquartile range 18-24 months). This follow-up included the time for recurrence or minimum of 18 months for patients with no recurrence. At 18 months, the success rates of AU with PSG and BMG were comparable $(89 \% \mathrm{v} / \mathrm{s} 91 \% ; p=0.70 ; 95 \%$ confidence interval (CI)0.33 to 5.21 ) and remained so over the duration of the study (Table S1). On Kaplan-Meier survival analysis (Fig. 2), we observed no statistically significant difference in restricturefree survival among the two groups (hazard ratio 1.39; $95 \%$ CI -0.48 to $4.03 ; p=0.53$ ). Based on the intention-to-treat population, the projected success rates were also comparable $[44 / 50(88 \%) \mathrm{v} / \mathrm{s} 42 / 50(84 \%) ; p=0.56 ; 95 \% \mathrm{CI}-0.45$ to 4.37] between the PSG and BMG arm at 18 months.

At 18 months, mean Qmax $(p=0.43)$, IPSS $(p=0.25)$, USS-PROM $(p=0.82)$ as well as improvements from baseline in Qmax $(p=0.06)$, IPSS $(p=0.43)$ and USS-PROM $(p=0.49)$ were comparable between the two arms. We observed no statistically significant difference in mean IIEF-Erectile domain ( $p=0.07)$, IIEF-Orgasmic domain $(p=0.11)$ and MSHQ-EJD $(p=0.20)$ at 18 months, between the two arms. (Table 2). Health-related quality of life domain
(EQ-5D-3L) of USS-PROM ( $p=0.33$ ) was not observed to be significantly different between the two arms at 18 months (Table 2). The treatment satisfaction domain of USS-PROM showed that $77 \%$ of patients in the BMG arm and $75 \%$ in the PSG arm were satisfied with the outcome.

Clavien-Dindo grade I treatment-emergent adverse events were seen in $12.7 \%$ and $16.7 \%$ patients of the PSG and BMG arms, respectively $(p=0.80)$. Out of the two patients with preputial oedema in the PSG arm, one patient underwent circumcision under local anaesthesia (Clavien-Dindo grade IIIa) for non-resolving preputial oedema. (Table S2).

\section{Discussion}

In non-comparative retrospective studies, the success rates of AU with PSG ranged from 71 to $86 \%$ and with BMG 89 to $96 \%$, respectively $[10,11]$. The results of comparative retrospective studies comparing the outcome of AU with BMG and PSG are inconsistent. On one hand, the outcome of BMG was found to be superior, and on the other hand, PSG was superior [12-14]. The prospective studies have shown either the superiority of BMG over PSG or comparable results with both, but all these studies are non-randomised (Table S3) [4, 15]. Therefore, the level of evidence 
Table 1 Baseline pre-operative and peri-operative characteristics of patients in the buccal mucosa graft (BMG) and inner preputial skin graft (IPG) groups

\begin{tabular}{lcc}
\hline Baseline variables & \multicolumn{2}{c}{ Study arms } \\
\cline { 2 - 3 } & BMG $(n=48)$ & IPG $(n=47)$ \\
\hline *Age (years) & $46(39-48)$ & $44(35-50)$ \\
*BMI $\left(\mathrm{kg} / \mathrm{m}^{2}\right)$ & $24.9(20.2-27.1)$ & $25.2(21.5-28.9)$ \\
*Duration (months) & $25(18-28)$ & $26(20-29)$ \\
*Length of stricture (mm) & $54(45-69)$ & $56(48-72)$ \\
Comparative site of stricture & & \\
Pendular & $5(10 \%)$ & $5(11 \%)$ \\
Bulbar & $10(21 \%)$ & $8(17 \%)$ \\
Peno-bulbar & $33(69 \%)$ & $34(72 \%)$ \\
Aetiology & & \\
Post-instrumentation & $37(77 \%)$ & $34(73 \%)$ \\
Post-inflammatory & $9(19 \%)$ & $10(21 \%)$ \\
Idiopathic & $2(4 \%)$ & $3(6 \%)$ \\
Pre-operative interventions & & \\
Dilatation & $28(58 \%)$ & $29(62 \%)$ \\
OIU & $9(19 \%)$ & $5(11 \%)$ \\
None & $11(22 \%)$ & $13(27 \%)$ \\
\#Operative time (minutes) & $155.6 \pm 19.7$ & $163.1 \pm 23.1$ \\
*Follow-up duration(months) & $22(18-24)$ & $23(18-24)$ \\
\hline
\end{tabular}

\# $($ Mean $\pm \mathrm{SD})$

*Median (interquartile range)

on the best option of graft for AU (BMG vs PSG) remains "low" and controversial [16].

Results from our prospective randomised study has shown similar 18 -month success rates (91\% vs $89 \% ; p=0.70)$ and comparable stricture-free survival (HR $1.39 ; p=0.534$ ) between the BMG and PSG arms. The heterogeneity in the reported success rates after AU can partly be attributed to lack of a uniform definition of success [17, 18], diverse aetiology [14], different surgical techniques[3, 19] and uneven sample size [14] (Table S3). Some have considered successful outcome, irrespective of secondary interventions for the recurrence [20]. The factors such as aetiology [16, 17], length and site of stricture [21] have been reported to affect the outcome of AU. But, these factors did not affect the success of AU on univariate analysis in our study (Table S4). The buccal mucosa graft can be used as dorsal, ventral or dorsal inlay and reported to have similar outcomes [22, 23]. However, comparative outcomes of penile skin graft used as ventral onlay or dorsal inlay is yet to be defined. For uniformity of the study, all patients in our study underwent dorsal onlay graft placement in both the groups.

Patient satisfaction after urethroplasty requires both, improvement in urinary flow and preservation of sexual function [5, 17]. Inclusion of patient-related outcome measures is a valuable tool in follow-up and enhances patient involvement and self-monitoring. Therefore, we have included functional parameters in our study to allow comprehensive assessment of post-operative outcome. We observed significant improvement in LUTS following AU, reflected by decrease in IPSS in both the arms. Raber et al. [4] also documented comparable improvements in IPSS scores after BMG and PSG substitution urethroplasty. Similar to our study, Vetterlein et al. [24] reported reasonable improvements in USS-PROM after BMG urethroplasty in radiation-induced strictures. Moreover, we found that the improvement across all domains of USS-PROM were comparable between the two study arms.
Fig. 2 Kaplan-Meier diagram showing comparative success between two arms; BMG-Buccal Mucosa Graft; IPG-Inner preputial graft

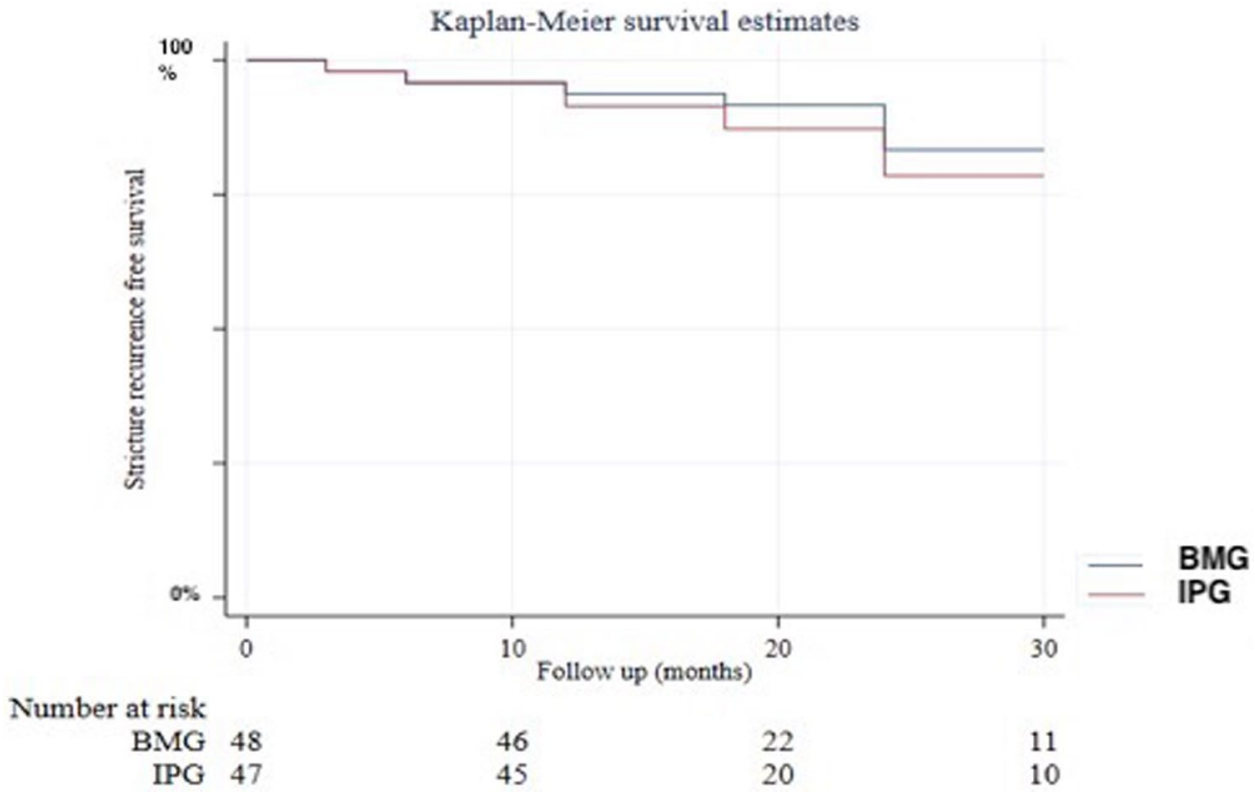


Table 2 Result of patients who did not require further treatment at different time intervals

\begin{tabular}{|c|c|c|c|c|c|c|}
\hline \multirow[t]{2}{*}{ Study arms } & \multirow[t]{2}{*}{ Pre-operative } & \multicolumn{4}{|l|}{ Follow-up } & \multirow{2}{*}{$\begin{array}{l}\text { Change from } \\
\text { pre-operative } \\
\text { values }\end{array}$} \\
\hline & & $\begin{array}{l}\text { At } 3 \text { m BMG } \\
(N=47) ; \text { IPG } \\
(N=46)\end{array}$ & $\begin{array}{l}\text { At } 6 \text { m BMG } \\
(N=46) ; \text { IPG } \\
(N=45)\end{array}$ & $\begin{array}{l}\text { At } 12 \mathrm{~m} \text { BMG } \\
(N=45) ; \mathrm{IPG} \\
(N=43)\end{array}$ & $\begin{array}{l}\text { At } 18 \mathrm{~m} \text { BMG } \\
(N=44) ; \text { IPG } \\
(N=42)\end{array}$ & \\
\hline & \multicolumn{6}{|c|}{$\mathrm{Qmax}(\mathrm{mL} / \mathrm{s})(\operatorname{mean} \pm \mathrm{SD})$} \\
\hline BMG & $8.10 \pm 1.61$ & $25.50 \pm 4.40$ & $23.95 \pm 5.61$ & $23.96 \pm 4.83$ & $23.93 \pm 3.83$ & $14.7 \pm 2.7$ \\
\hline IPG & $7.91 \pm 1.35$ & $25.15 \pm 4.52$ & $23.44 \pm 3.40$ & $22.10 \pm 5.19$ & $21.00 \pm 5.00$ & $13.3 \pm 3.3$ \\
\hline \multirow[t]{2}{*}{$p$ value } & 0.31 & 0.095 & 0.17 & 0.64 & 0.43 & 0.06 \\
\hline & \multicolumn{6}{|c|}{ IPSS (mean \pm SD) } \\
\hline BMG & $15.6 \pm 5.11$ & $4.18 \pm 1.20$ & $3.33 \pm 1.06$ & $3.21 \pm 0.74$ & $2.75 \pm 0.93$ & $12.8 \pm 2.4$ \\
\hline IPG & $15.7 \pm 4.03$ & $3.71 \pm 1.08$ & $3.76 \pm 1.45$ & $3.42 \pm 1.18$ & $3.57 \pm 1.78$ & $12.3 \pm 2.3$ \\
\hline \multirow[t]{2}{*}{$p$ value } & 0.13 & 0.69 & 0.61 & 0.49 & 0.25 & 0.43 \\
\hline & \multicolumn{6}{|c|}{ IIEF-ED $($ mean \pm SD) } \\
\hline BMG & $16.7 \pm 6.70$ & $17.1 \pm 6.91$ & $19.2 \pm 4.03$ & $19.4 \pm 3.92$ & $19.6 \pm 3.68$ & $2.96 \pm 0.32$ \\
\hline IPG & $19.6 \pm 5.40$ & $19.7 \pm 5.42$ & $20.4 \pm 4.53$ & $20.7 \pm 2.44$ & $20.9 \pm 2.36$ & $1.10 \pm 0.41$ \\
\hline \multirow[t]{2}{*}{$p$ value } & 0.06 & 0.06 & 0.53 & 0.16 & 0.07 & 0.09 \\
\hline & \multicolumn{6}{|c|}{ IIEF-OD (mean \pm SD) } \\
\hline BMG & $5.56 \pm 2.52$ & $5.62 \pm 2.44$ & $6.33 \pm 3.39$ & $6.39 \pm 2.15$ & $6.43 \pm 2.27$ & $1.21 \pm 0.5$ \\
\hline IPG & $6.71 \pm 2.42$ & $6.56 \pm 2.51$ & $7.19 \pm 1.86$ & $7.28 \pm 1.25$ & $7.21 \pm 1.31$ & $0.9 \pm 0.02$ \\
\hline \multirow[t]{2}{*}{$p$ value } & 0.23 & 0.58 & 0.09 & 0.19 & 0.11 & 0.07 \\
\hline & \multicolumn{6}{|c|}{ MSHQ-EJD (mean \pm SD) } \\
\hline BMG & $11.9 \pm 5.16$ & $12.1 \pm 5.14$ & $13.8 \pm 4.54$ & $13.9 \pm 3.92$ & $13.1 \pm 4.97$ & $1.17 \pm 0.4$ \\
\hline IPG & $13.7 \pm 5.24$ & $13.0 \pm 5.11$ & $14.6 \pm 4.10$ & $14.8 \pm 3.13$ & $14.6 \pm 3.03$ & $1.11 \pm 0.22$ \\
\hline \multirow[t]{2}{*}{$\mathrm{p}$ value } & 0.50 & 0.48 & 0.12 & 0.31 & 0.20 & 0.46 \\
\hline & \multicolumn{6}{|c|}{ USS-PROM: LUTS domain (mean \pm SD) } \\
\hline BMG & $14.6 \pm 5.90$ & $5.11 \pm 1.94$ & $5.19 \pm 1.75$ & $5.47 \pm 1.32$ & $5.53 \pm 1.99$ & $9.83 \pm 2.23$ \\
\hline IPG & $15.0 \pm 5.01$ & $4.83 \pm 1.81$ & $4.90 \pm 1.93$ & $5.21 \pm 1.76$ & $5.44 \pm 1.87$ & $10.12 \pm 1.92$ \\
\hline \multirow[t]{2}{*}{$p$ value } & 0.70 & 0.46 & 0.44 & 0.41 & 0.82 & 0.49 \\
\hline & \multicolumn{6}{|c|}{ USS-PROM: peeling voiding score $($ mean $\pm \mathrm{SD})$} \\
\hline BMG & $3.81 \pm 0.71$ & $1.22 \pm 0.41$ & $1.44 \pm 0.62$ & $1.53 \pm 0.91$ & $1.91 \pm 0.83$ & $1.98 \pm 0.43$ \\
\hline IPG & $3.65 \pm 0.66$ & $1.29 \pm 0.56$ & $1.39 \pm 0.86$ & $1.61 \pm 0.77$ & $1.98 \pm 0.97$ & $1.81 \pm 0.52$ \\
\hline \multirow[t]{2}{*}{$p$ value } & 0.25 & 0.48 & 0.74 & 0.64 & 0.70 & 0.08 \\
\hline & \multicolumn{6}{|c|}{ USS-PROM: EQ-5D-3L index score $($ mean $\pm S D)$} \\
\hline BMG & $0.51 \pm 0.13$ & $0.95 \pm 0.21$ & $0.91 \pm 0.23$ & $0.89 \pm 0.20$ & $0.86 \pm 0.21$ & $0.41 \pm 0.11$ \\
\hline IPG & $0.45 \pm 0.19$ & $0.97 \pm 0.16$ & $0.93 \pm 0.21$ & $0.92 \pm 0.17$ & $0.90 \pm 0.19$ & $0.44 \pm 0.16$ \\
\hline$p$ value & 0.07 & 0.60 & 0.65 & 0.43 & 0.33 & 0.29 \\
\hline
\end{tabular}

The surgical technique of $\mathrm{AU}$ is not likely to cause any damage or compromise to the vascular or neural or cavernosal components of erection. Ejaculatory function may either improve due to relief of obstruction or deteriorate due to division or traction injury to the bulbospongiosus muscle or perineal nerves during urethroplasty. The incidence of sexual dysfunction following anterior urethroplasty has been reported to be 0-38\% [25]. Coursey et al. reported that even circumcision can be associated with erectile dysfunction (ED) in 27\% [26]. Therefore, ED following AU could be psychogenic rather than organic. In our study, there was no significant change in IIEF-ED, IIEF-OD and MSHQ-EJD scores at 18 months in both the arms compared to pre-operative scores. Raber et al. [4] also reported that IIEF-ED scores were unaffected in both the arms. However in their study, IIEF-OD improved significantly with penile skin graft as compared to BMG, but no plausible explanation was given for this observation.

In patients with lengthier strictures requiring BMG harvest from bilateral cheeks, oral rehabilitation is likely to take a longer time. Ibrahim et al. reported that as compared to unilateral, bilateral BMG harvest patients have higher pain scores in the immediate post-operative period, along with significantly longer duration to return to normal diet and to attain optimal mouth opening [27]. The inner prepuce yields a wider graft, which can be divided to achieve two grafts of equal length 
for substituting longer defect without increasing the donor site morbidity. In our study, perioral numbness was seen in one (2.7\%) and fibrosis at donor site with restriction of mouth opening was observed in two (4.1\%) patients. Interestingly, both patients with fibrosis were using artificial dentures.

Amidst the prevailing COVID-19 pandemic, numerous recommendations and guidelines have been proposed for safety as well as feasibility of surgical and interventional procedures. However, none of them have addressed the safety of BMG for AU. SARS-CoV-2 RNA is present in saliva of up to $91 \%$ of COVID-19 patients [28] and harvesting BMG could pose a potential risk of cross infection between patients and health-care workers. Therefore, PSG should be preferred in such situation due to its relative safety with equivalent success, because it can be done under regional anaesthesia and the surgical field remains confined to the perineum only.

Complications like bleeding, wound infection, urinary tract infections and post-void dribbling are reported with PSG harvesting [29]. In our study, preputial oedema at the donor site in the PSG arm was observed in two patients. It resolved with conservative measures in one patient within 3 months and circumcision was needed in one patient for non-resolving preputial oedema. When only a part of preputial skin is harvested, the surgeon intends to leave the rest of the skin with the apprehension of future need. This skin tag may become oedematous and disfiguring, which happened in two of our initial cases. Subsequently, we practised circumcision during PSG harvest and did not observe preputial oedema in any patient.

The prospective randomisation is the strength of this study. This is the first randomised controlled trial comparing AU with BMG and PSG for the treatment of long segment anterior urethral strictures. The outcome of AU was evaluated not only in terms of voiding (IPSS, UFM and calibration), but also for sexual function (erection, orgasmic and ejaculatory domains). The limitation of the study is the short follow-up and outcome assessment at 18 months. The objective assessment with urethrogram or cystoscopy was performed only when deemed necessary and we acknowledge that all patients with abnormal uroflow had unsuccessful calibration with 16 French foley catheter or unsuccessful endoscopy with 17 French cystoscope. This study remained non-blinded because the different graft site could be difficult to camouflage. Though the follow-up duration is suboptimal, it should be scrutinized with due weightage as a practical limitation in a randomized study on urethral reconstruction [30].

\section{Conclusions}

In this study, we found no statistical significant difference in the outcomes of AU using BMG or PSG. However, patients undergoing BMG substitution may need to be counselled, especially for oral morbidity and rehabilitation in the early post-operative period.

Supplementary Information The online version contains supplementary material available at https://doi.org/10.1007/s00345-021-03843-x.

Funding None.

\section{Declarations}

Conflicts of interest None.

Ethical approval PGIMER Ethics committee approval was taken, Ref no. INT/EC/2018/000909.

Consent to participate Written informed consent was taken from all patients.

Consent for publication Consent was obtained.

\section{References}

1. Mangera A, Chapple C (2010) Management of anterior urethral stricture: an evidence-based approach. Curr Opin Urol 20:453-458

2. Lumen N, Oosterlinck W, Hoebeke P (2012) Urethral reconstruction using buccal mucosa or penile skin grafts: systematic review and meta-analysis. Urol Int 89(4):387-394

3. Barbagli G, Guazzoni G, Lazzeri M (2008) One- stage bulbar urethroplasty: retrospective analysis of the results in 375 patients. Eur Urol 53:828-833

4. Raber M, Naspro R, Scapaticci E et al (2005) Dorsal onlay graft urethroplasty using penile skin or buccal mucosa for repair of bulbar urethral stricture: results of a prospective single center study. Eur Urol 48(6):1013-1017

5. Bertrand LA, Voelzke BB, Elliott SP et al (2016) Measuring and predicting patient dissatisfaction after anterior urethroplasty using patient reported outcomes measures. J Urol 196(2):453-461

6. Jackson MJ, Chaudhury I, Mangera A et al (2013) A prospective patient-centred evaluation of urethroplasty for anterior urethral stricture using a validated patient-reported outcome measure. Eur Urol 64(5):777-782

7. Peeling WB (1989) Diagnostic assessment of benign prostatic hyperplasia. Prostate Suppl 2:51-68

8. EuroQol Group (1990) EuroQol-a new facility for the measurement of health-related quality of life. Health Policy 16(3):199-208

9. Kulkarni S, Barbagli G, Sansalone S et al (2009) One-sided anterior urethroplasty: a new dorsal onlay graft technique. BJU Int 104(8):1150-1155

10. Tyagi S, Parmar K, Sharma A, Devana S, Kumar S, Singh SK, Bora G, Mavuduru RM (2021) Dorsolateral Penile skin graft urethroplasty for anterior urethral strictures: long-term outcomes from a single tertiary care centre. World J Urol 13:1-6 
11. Kane CJ, Tarman GJ, Summerton DJ et al (2002) Multi-institutional experience with buccal mucosa onlay urethroplasty for bulbar urethral reconstruction. J Urol 167:1314-1317

12. Barbagli G, Kulkarni SB, Fossati N et al (2014) Long-term follow up and deterioration rate of anterior substitution urethroplasty. $\mathrm{J}$ Urol 192:808-813

13. Berger AP, Deibl M, Bartsch G et al (2005) A comparison of onestage procedures for post- traumatic urethral stricture repair. BJU Int 95:1299-1302

14. Lumen N, Browaeys $H$, Hoebeke $P$ et al (2008) Ventral onlay graft urethroplasty using genital skin or buccal mucosa in the treatment of bulbar strictures: a retrospective analysis of 41 cases. Curr Urol 2:10-15

15. Lumen N, Hoebeke P, Lumen $N$ et al (2010) Urethroplasty for urethral strictures: quality assessment of an in-home algorithm: original article: clinical investigation. Int J Urol 17:167-174

16. Sharma G, Sharma S, Parmar K (2020) Buccal mucosa or penile skin for substitution urethroplasty: a systematic review and metaanalysis. Indian J Urol 36:81-88

17. Erickson BA, Ghareeb GM (2017) Definition of successful treatment and optimal follow-up after urethral reconstruction for urethral stricture disease. Urol Clin North Am 44:1-9

18. Payne SR, Fowler S, Mundy AR (2020) Analysis of a 7-year national online audit of the management of open reconstructive urethral surgery in men. BJU Int 125(2):304-313

19. D'hulst P, Muilwijk T, Vander Eeckt K, et al (2020) Patientreported outcomes after buccal mucosal graft urethroplasty for bulbar urethral strictures: results of a prospective single-centre cohort study. BJU International 126(6):684-693

20. Elliot SP, Metro MJ, McAninch JW (2003) Long-term follow-up of the ventrally placed buccal mucosa onlay graft in bulbar urethral reconstruction. J Urol 169:1754-1757

21. Wang K, Miao X, Wang L et al (2009) Dorsal onlay versus ventral onlay urethroplasty for anterior urethral stricture: A meta-analysis. Urol Int 83:342-348
22. Vasudeva P, Nanda B, Kumar A et al (2015) Dorsal versus ventral onlay buccal mucosal graft urethroplasty for long-segment bulbar urethral stricture: A prospective randomized study. Int J Urol 22(10):967-971

23. Singh SK, Agrawal SK, Mavuduru RS (2011) Management of the stricture of fossa navicularis and pendulous urethral strictures. Indian J Urol 27(3):371-7

24. Vetterlein MW, Kluth LA, Zumstein V et al (2020) Buccal mucosal graft urethroplasty for radiation-induced urethral strictures: an evaluation using the extended Urethral Stricture Surgery Patient-Reported Outcome Measure (USS PROM). World J Urol 38:2863-2872

25. Blaschko SD, Sanford MT, Cinman NM et al (2013) De novo erectile dysfunction after anterior urethroplasty: a systematic review and meta-analysis. BJU Int 112:655-663

26. Coursey JW, Morey AF, McAninch JW et al (2001) Erectile function after anterior urethroplasty. J Urol 166:2273-2276

27. Bozkurt İH, Yalçınkaya F, Sertçelik MN et al (2013) Comparison of uni-and bilateral buccal mucosa harvesting in terms of oral morbidity. Turk J Urol 39(1):43-47

28. Wang To KK, Tsang OT, Yip CC et al (2020) Consistent detection of 2019 novel coronavirus in saliva. Clin Inf Dis 71(15):841-843

29. Hussein MM, Almogazy H, Mamdouh A et al (2016) Urethroplasty for treatment of long anterior urethral stricture: buccal mucosa graft versus penile skin graft-does the stricture length matter? Int Urol Nephrol 48:1831-1835

30. Barbagli G, Lazzeri M (2008) Can reconstructive urethral surgery proceed without randomised controlled trials? Eur Urol 54:709-711

Publisher's Note Springer Nature remains neutral with regard to jurisdictional claims in published maps and institutional affiliations. 